\title{
Optimal Design of Support for Planoconvex Quartz Resonator
}

\author{
Jing $\mathrm{Ji}^{1}{ }^{*}$ Meng Zhao, ${ }^{1}$ Hiroshi Oigawa, ${ }^{2}$ and Toshitsugu Ueda ${ }^{2}$ \\ ${ }^{1}$ School of Electro-Mechanical Engineering, Xidian University, No. 2 South Taibai Road, Xi'an 710071, P.R. China \\ ${ }^{2}$ IPS Research Center, Waseda University, 2-2 Hibikino, Wakamatsu-ku, Kitakyushu, Fukuoka 808-0135, Japan
}

(Received December 1, 2017; accepted May 9, 2018)

Keywords: quartz resonator, planoconvex, optimal design, support, energy trapping

In this paper, we report the optimal design of a support for a fundamental planoconvex ATcut quartz resonator fabricated using our newly developed etching process. We established a three-dimensional finite element model and carried out eigen-frequency analysis. To describe quantitatively the energy trapping performance of the resonator, we introduced a parameter called the energy trapping rate, which is defined by the ratio of the vibration energy inside to that outside of the electrode region. Two types of our new designs were evaluated in this work, and the optimal one for providing a large energy-trapping rate was determined. On the basis of the calculated results, we fabricated resonators and measured their $Q$-factors. The calculated and experimental results show an improvement in the efficiency of resonator performance. High $Q$-factors were also obtained.

\section{Introduction}

Currently, high-frequency AT-cut quartz resonators with high performance are in increasing demand for use in frequency generators and frequency control devices in telecommunication systems. The performance of the high-frequency quartz resonators is mainly influenced by energy trapping and the decoupling characteristic. ${ }^{(1)}$ Upon electrode mass loading, the resonance frequency of the excitation electrode region is lower than that of other regions. ${ }^{(2)}$ This results in the thickness-shear (TS) vibration mode being excited in the excitation electrode region, and the corresponding vibration decays exponentially outside. To achieve a high $Q$-factor for a high-frequency resonator, the resonator structure should be optimized to confine vibration energy in the excitation electrode region efficiently. On the other hand, ideally, highfrequency TS resonators vibrate in the pure fundamental TS mode. However, because of the boundary and the elastic constant of quartz, the fundamental TS mode inevitably couples with unwanted vibration modes. ${ }^{(3)}$ To achieve a high $Q$-factor for resonators, the resonator structure should be optimized to reduce unwanted mode couplings.

Dimensions seriously affect energy trapping and vibration coupling of resonators. Ever since Mindlin et al. began to optimize the rectangular resonator applying plate theory, ${ }^{(4-7)}$ many research works have sought to determine the optimal excitation electrode shape and size

*Corresponding author: e-mail: jingji@xidian.edu.cn http://dx.doi.org/10.18494/SAM.2018.1832 
to improve energy trapping ${ }^{(8-10)}$ and to find the optimal excitation electrode size to improve decoupling. ${ }^{(11,12)}$ Many theories on new and different shapes of excitation electrodes, including convex, circular, and nonuniform thickness, have been published. Recently, there has been growing research interest in using FEM programs to study the effect of electrodes. Many efficient results of electrode optimization have been obtained using $2 \mathrm{D} \mathrm{FEM}^{(13,14)}$ and 3D FEM. ${ }^{(15-17)}$

Besides the vibration portion, the support structure is another factor seriously affecting energy trapping and vibration coupling of resonators. Current research of support mainly discussed the effect on energy loss, which is a result of stress waves carrying energy away from the vibration portion of the resonator. Many rewarding approaches for reducing the transmission of energy to the substrate, such as selecting a good vibration mode ${ }^{(18)}$ and using special structural designs, ${ }^{(19,20)}$ have been proposed.

However, previous work still leaves us with the following situation. 1) Studies have focused on overtone-type resonators, which are not as good as fundamental-type resonators. 2) The optimization of the supports to improve energy trapping and decoupling performance have rarely been discussed.

In 2013, we reported work on support optimization for a fundamental-type rectangular quartz resonator. ${ }^{(21)}$ Because a convex quartz resonator can confine vibrational energy more efficiently than a rectangular quartz resonator, the convex quartz resonators, including planoconvex and biconvex resonators, can achieve higher $Q$-factors than resonators with other shapes. ${ }^{(22)}$ To further improve the performance of a resonator, we present herein new research findings regarding support optimization for a high-frequency planoconvex quartz resonator fabricated by our new etching process.

Two kinds of support structures were designed and discussed in this work: one is a cantilever type, the other a mesa type. The cantilever-type structure is shown in top, bottom, and sectional views in Fig. 1. The quartz substrate (shown in blue) consists of two parts: a

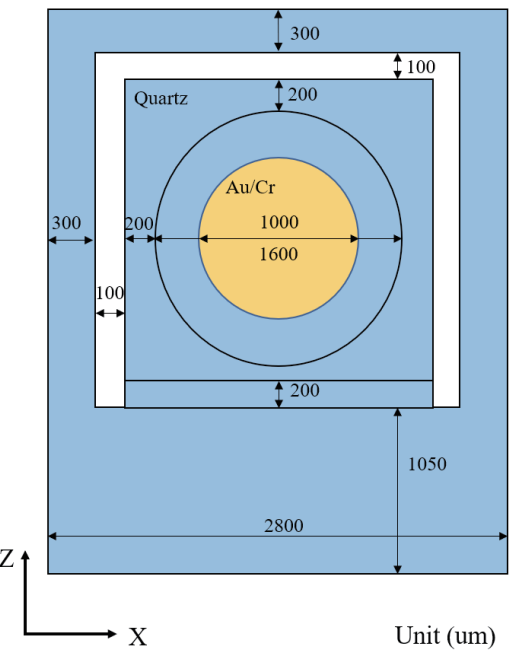

(a)

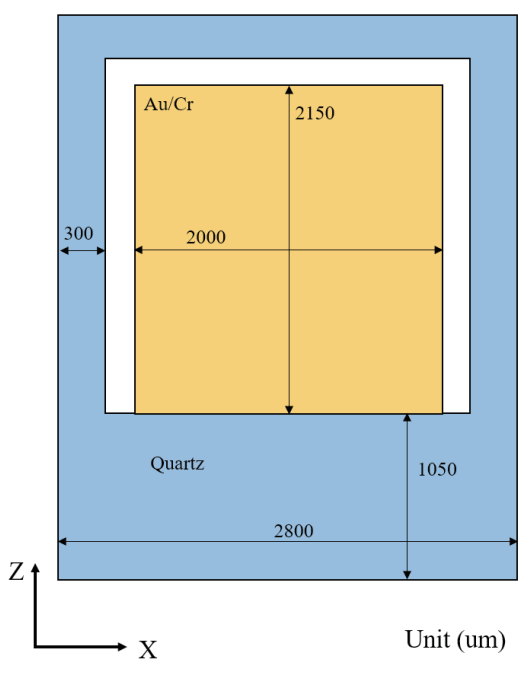

(b)

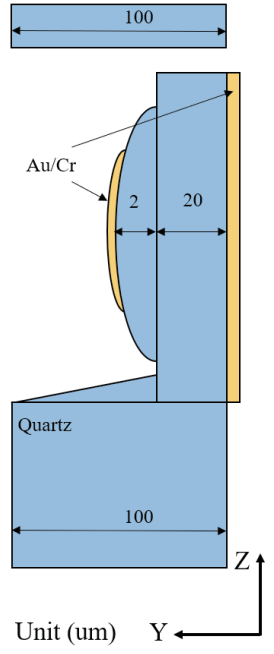

(c)

Fig. 1. (Color online) Profile of resonator structure with cantilever-type support. (a) Top view, (b) bottom view, and (c) sectional view. 
planoconvex vibration portion and a support. The vibration portion is partially covered by an upper planoconvex excitation electrode and fully covered by a lower rectangular electrode connected to the ground. The electrodes are $\mathrm{Cr} / \mathrm{Au}$ two-layer types (shown in yellow). The central vibration part is partially separated from the support. The mesa-type structure is also shown in top, bottom, and sectional views in Fig. 2. The quartz substrate consists of two parts: a central planoconvex vibration portion and a surrounding support. The central vibration part is connected with the support, but they have a height difference.

\section{Optimization}

The material constants that we used came from IEEE Standard on Piezoelectricity and materials library of COMSOL MULTIPHYSICSTM.

The elastic constant of AT-cut quartz is

$$
\boldsymbol{C}_{i j}^{E}=\left[\begin{array}{cccccc}
86.74 & -8.25 & 27.15 & -3.66 & 0 & 0 \\
-8.25 & 129.77 & -7.42 & 5.7 & 0 & 0 \\
27.15 & -7.42 & 102.83 & 9.92 & 0 & 0 \\
-3.66 & 5.7 & 9.92 & 38.61 & 0 & 0 \\
0 & 0 & 0 & 0 & 68.81 & 2.53 \\
0 & 0 & 0 & 0 & 2.53 & 29.01
\end{array}\right] \times 10^{9}(\mathrm{~Pa}) .
$$

The piezoelectric constant and dielectric constant matrix for AT-cut quartz are

$$
\boldsymbol{e}=\left[\begin{array}{cccccc}
-0.171 & 0.152 & 0.0187 & -0.067 & 0 & 0 \\
0 & 0 & 0 & 0 & -0.108 & 0.095 \\
0 & 0 & 0 & 0 & 2.53 & 29.01
\end{array}\right]\left(\mathrm{C} / \mathrm{m}^{2}\right)
$$

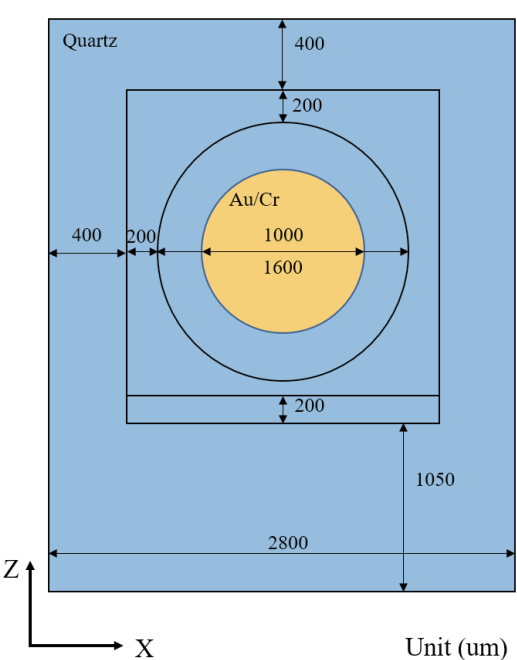

(a)

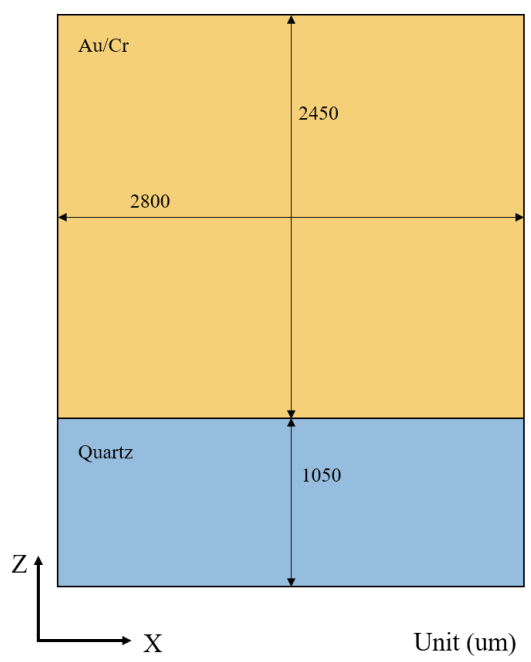

(b)

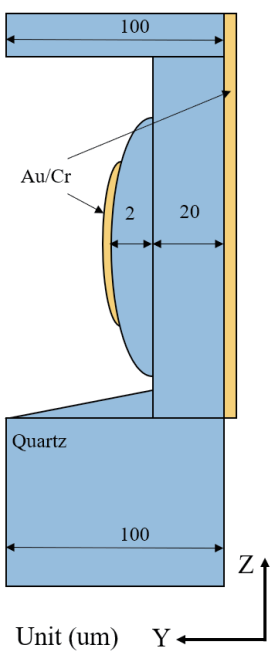

(c)

Fig. 2. (Color online) Profile of the resonator structure with mesa-type support. (a) Top view, (b) bottom view, and (c) sectional view. 


$$
\boldsymbol{\varepsilon}=\left[\begin{array}{ccc}
39.21 & 0 & 0 \\
0 & 39.82 & 0.86 \\
0 & 0.86 & 40.42
\end{array}\right] \times 10^{-12}(\mathrm{~F} / \mathrm{m})
$$

The parameters of the Au and $\mathrm{Cr}$ electrodes are shown in Table 1. The constraint condition in the boundary setting is free.

Firstly, we carry out an eigenfrequency analysis using linear free triangular elements to establish 3D finite element modeling. The degrees of freedom are approximately 400000. The meshing mode of a resonator with cantilever-type support is shown in Fig. 3.

Secondly, the main fundamental TS mode was selected from calculated vibration modes including spurious ones. By drawing the displacement distributions for two kinds of support structure, the energy trapping rate and decoupling performance were examined.

To achieve a high $Q$-factor, the dimensions of a resonator's support should be optimized to confine vibration energy to the excitation electrode region. To quantitatively describe the energy trapping performance for these cases, an energy trapping rate was introduced:

$$
R=\frac{E_{e}}{E_{e}+E_{o}}
$$

where $E_{e}$ denotes the vibration energy in the electrode region and $E_{o}$ denotes the vibration energy outside in the fundamental TS mode.

Table 1

Parameters of electrodes.

\begin{tabular}{lcc}
\hline Parameter & $\mathrm{Cr}$ & $\mathrm{Au}$ \\
\hline Density $\left(\mathrm{kg} / \mathrm{m}^{3}\right)$ & $7.19 \times 10^{3}$ & $19.30 \times 10^{3}$ \\
Young's modulus $(\mathrm{GPa})$ & 279 & 79 \\
Poisson's ratio & 0.21 & 0.42 \\
\hline
\end{tabular}

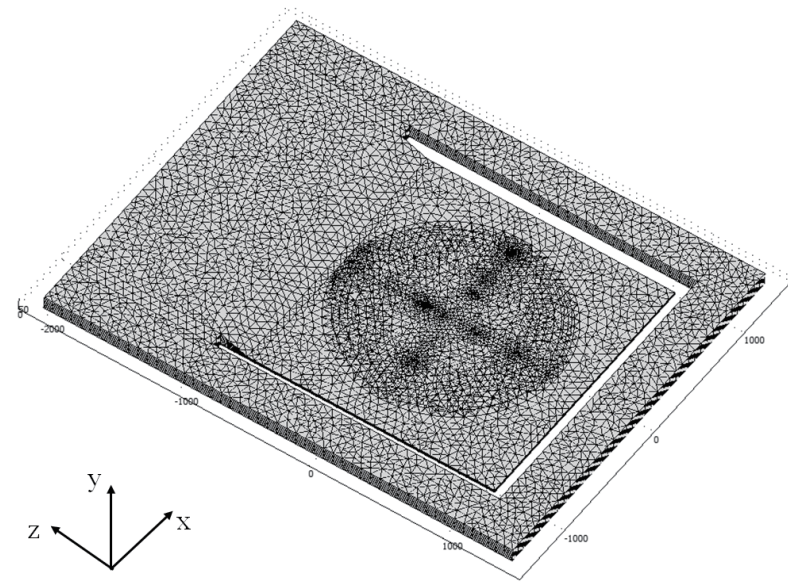

Fig. 3. Finite element modeling of cantilever-type support design. 
The calculated energy trapping rate for the cantilever-type support design was $87.1 \%$ compared with $20.36 \%$ for the mesa-type support design; the improvement is significant.

The energy trapping performance was affected by the dimensions of the support because of energy transmission. For the cantilever-type design, although the vibration part is separated from the support, energy loss in the central excitation electrode should be decreased. Figure 4 gives the $x$-displacement surface distribution in the fundamental TS mode for resonators with cantilever-type and mesa-type support. To enable a clear comparison, the $x$-displacement has been normalized. From the normalized $x$-displacement surface distribution, we can find that the cantilever-type design gives a smoother (less spurious vibration) and more concentrated vibration energy distribution (better energy trapping performance).

\section{Verification}

According to the calculated results, for the cantilever-type resonator, vibrational energy was much more efficiently confined in the central excitation region of the electrode than in the mesa-type design, and with less spurious vibration. On the basis of these results, we fabricated resonators including cantilever-type and mesa-type support structures (shown in Fig. 5), as well as rectangular resonators.

We developed a new fabrication method for planoconvex electrodes in this work, which is an improved version of the traditional dry etching method. Our method comprises reflow of a thinner steam, deep reactive ion etching (DRIE), and wet etching. Firstly, we spin coat the photoresist on the surface of a 100- $\mu \mathrm{m}$-thick quartz wafer. After exposure and development, photoresist remains only in the convex region. Secondly, reflow of a thinner stream is performed. By blowing nitrogen gas containing a thinner stream onto the thick film photoresist, the photoresist is softened and becomes fluid. Then the shape of photoresist film changes to a convex lens shape because of surface tension. Thereafter, the softened resist is stiffened by

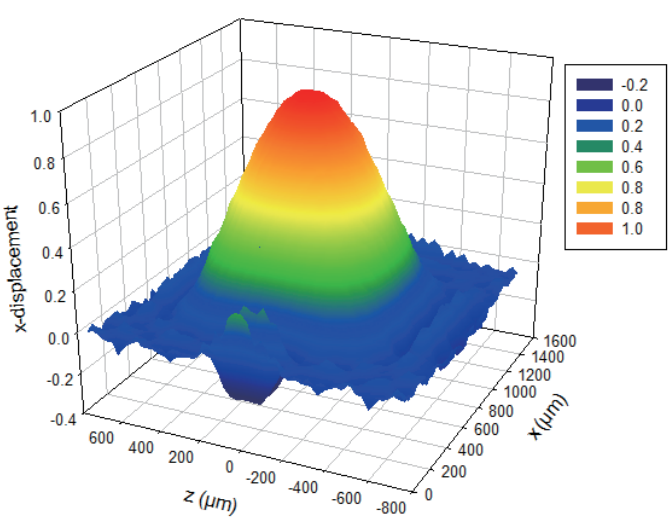

(a)

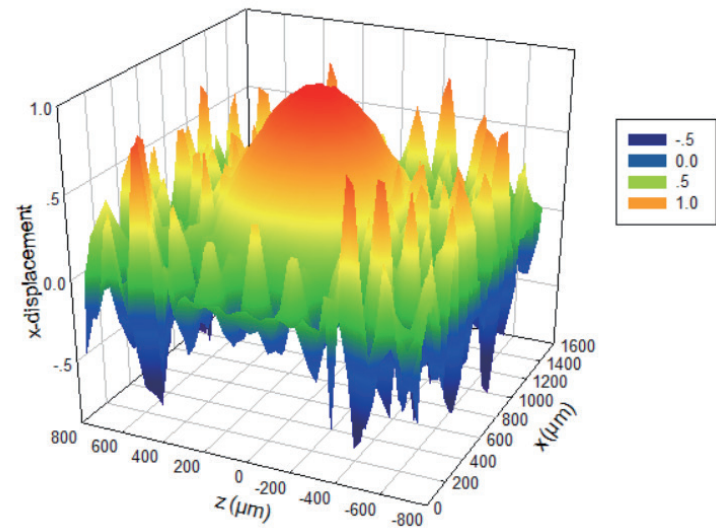

(b)

Fig. 4. (Color online) Normalized $x$-displacement surface distribution for two kinds of support structure in the fundamental TS mode. (a) Cantilever type and (b) mesa type. 


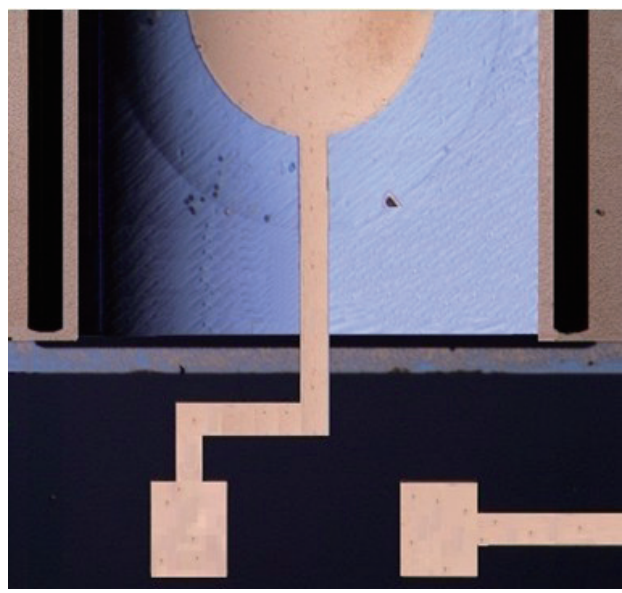

(a)

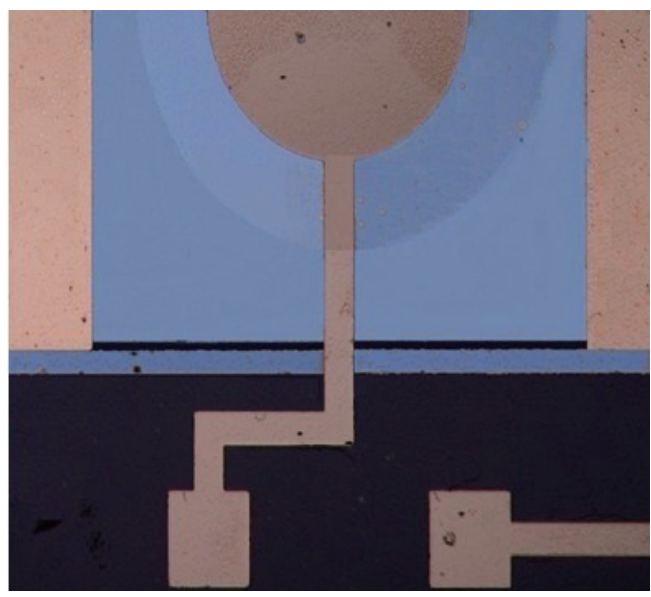

(b)

Fig. 5. (Color online) Optical micrographs of the fabricated resonators: (a) cantilever-type support design and (b) mesa-type support design.

drying and baking. Thirdly, DRIE is performed under the following processing conditions: process gas $\mathrm{SF}_{6}: \mathrm{C}_{4} \mathrm{~F}_{8} 80 \%$, pressure $0.26 \mathrm{~Pa}$, $\mathrm{RF}$ power $100 \mathrm{~W}$, and processing time up to $5 \mathrm{~min}$. Finally, the external shapes of the quartz and electrode are wet-etched.

The measurement system we used comprises the impedance analyzer (Agilent 4396B, 100 $\mathrm{kHz}$ to $3 \mathrm{GHz}$ ), the transmission/reflection test set (87512A, DC to $2 \mathrm{GHz}$ ), the high-frequency probe (ESST coaxial probe arm A-C1, DC to $3 \mathrm{GHz}$ ), and the manual prober. Before measuring the $Q$-factor of fabricated resonators, we examined frequency resolution. Using the span value to represent the measurement range, for a $10 \mathrm{MHz}$ span, the frequency resolution is 12.5 $\mathrm{kHz}$; for $100 \mathrm{kHz}$, the frequency resolution is $125 \mathrm{~Hz}$. For example, if the $Q$-factor is about 10000 , the half-width of the peak $\Delta f$ can be calculated by the following equation with $10 \mathrm{kHz}$. Therefore, the $10 \mathrm{MHz}$ span is insufficient for measurement accuracy, but $100 \mathrm{kHz}$ span is enough.

$$
Q=\frac{f_{0}}{\Delta f}=\frac{f_{0}}{f_{+}-f_{-}}
$$

Using the frequency response results, we calculated the $Q$-factors for all our fabricated resonators. All the $Q$-factor values of our fabricated resonators are shown in Table 2. From the frequency response, we can also find that spurious vibration is far away from the main TS mode. From table 2, we see that the average measured $Q$-factors were 17455 for the mesa-type and 18291 for the cantilever-type. The largest $Q$-factors achieved were 39090 for the mesa-type and 45682 for the cantilever-type. Compared with rectangular quartz resonators, which have average $Q$-factors of about 7604 and the largest $Q$-factor of about 17817, the two newly designed planoconvex resonators demonstrated improved performance. Moreover, we found that the cantilever-type structure is the optimal support design and has better $Q$-factors than those of the mesa-type structure. 
Table 2

Measured $Q$-factors of fabricated resonators.

\begin{tabular}{lccc}
\hline & Rectangular resonators & $\begin{array}{c}\text { Planoconvex resonators } \\
\text { with cantilever-type support }\end{array}$ & $\begin{array}{c}\text { Planoconvex resonators } \\
\text { with mesa-type support }\end{array}$ \\
\hline Samples & 36 & 19 & 19 \\
Average frequency $(\mathrm{MHz})$ & 90.6 & 86.6 & 87.1 \\
Average $Q$-factor & 7604 & 18291 & 17455 \\
Maximum $Q$-factor & 17817 & 45682 & 39090 \\
\hline
\end{tabular}

\section{Conclusions}

In this work, we reported an optimal design for the support of a fundamental planoconvex AT-cut quartz resonator. We concluded that the first improvement was to change our former rectangular resonators into planoconvex ones. The second improvement was the determination of the optimal support structure. Two kinds of support structure were designed and discussed. A three-dimensional finite element model was established to carry out eigen-frequency analysis. a parameter named the energy trapping rate was introduced to describe quantitatively the energy trapping performance of a resonator. From the calculated results, the optimal resonator with a large energy trapping rate and less spurious vibration was determined. Using the simulation results, we fabricated resonators and measured their $Q$-factors. The cantilevertype support design showed significant improvement over the mesa-type. Our optimization work was validated in this study.

The originality of this research can be concluded as follows. 1) The optimal design is based on AT-cut quartz MEMS resonators with a high frequency value realized by the fundamental TS vibration mode. 2) For the optimal design of the AT-cut high-frequency quartz resonators, the influence of the support on improving the energy trapping effect and reducing spurious couplings was examined. The optimal design of resonators simultaneously realized the improvement of the energy trapping effect and the reduction of spurious couplings. To quantitatively describe the energy trapping performance of the resonator, we defined an original parameter named the energy trapping rate. 3) Two kinds of support structure were designed and evaluated. The optimal one that provides a large energy trapping rate and less spurious vibration was determined. Measured $Q$-factors of the fabricated resonators indicated an improvement in the efficiency of resonator performance.

However, in this work, we mainly focused on studying the influence of the support on the resonator performance on the basis of the prespecified dimensions of the vibrational portion. If the dimensions of the planoconvex vibrational part, including the upper excitation electrode, lower grounded electrode, and convex quartz part, are changed, the energy trapping performance also changes. However, the optimization method in this work should be adaptable to changes in the dimensions of the vibrational portion. Moreover, to ensure good performance of planoconvex resonators, the optimal support structure should be determined by examining spurious couplings. In the future, we will further focus on how to reduce coupling for different kinds of spurious vibrations. 
Moreover, in the simulation process, for a different packaging method, the constraint conditions changed. In this work, we measured the performance of resonators before they were packaged. Therefore, the base surface was not fixed to the substrate. Strictly speaking, the fixed area partially appeared where we set pins or leads. The remaining parts of the resonators were connected to nothing. This is very similar to the idealized conditions of free setting. At the same time, in order to carry out simulation efficiently and save calculation time, the structure of resonators was simplified to some degree. For example, the pins and leads were not considered in the current work. We will further focus these discussions in our future work.

\section{Acknowledgments}

This work was supported by the National Natural Science Foundation of China (51702251) and Fundamental Research Funds for the Central Universities (XJS16064).

\section{References}

1 C. J. Wilson: J. Phys. D: Appl. Phys. 7 (1974) 2450

2 S. Seto, S. Horiuchi, and K. Yamada: Jpn. J. Appl. Phys. 49 (2010) 07HC05.

3 J. Wang, Q. Pan, L. Yang, and M. Chao: Proc. 2010 IEEE Int. Ultrasonics Symp. (IEEE, 2010) 1246.

4 R. D. Mindlin and P. C. Y. Lee: Int. J. Solids Struct. 2 (1966) 125.

5 R. J. Byrne, P. Lloyd, and W. J. Spencer: J. Acoust. Soc. Am. 43 (1966) 232.

6 J. L. Bleustein and H. F. Tiersten: J. Acoust. Soc. Am. 43 (1968) 1311.

7 Y. K. Yong, J. T. Stewart, J. Detaint, A. Zarka, and B. Capelle: IEEE Trans. Ultrason. Ferroelectr. Freq. Control 39 (1992) 609.

8 K. Nakamura and H. Shimizu: Proc. 1976 Ultrasonics Symp. (1976) 606-609.

9 K. Nakamura and R. Yasuike: Proc. 1990 Frequency Control 44th Annu. Symp. (IEEE, 1990) 372-377.

10 M. Onoe: Proc. 2005 IEEE Frequency Control Symp. Exposition (IEEE, 2005) 433-441.

11 J. Wang: IEEE Trans. Ultrason. Ferroelectr. Freq. Control 52 (2005) 2023.

12 Z. Yang, S. Guo, and J. Yang: Proc. 2008 IEEE Ultrasonics Symp. (IEEE, 2008) 2158-2160.

13 J. Wang and L. Shen: Proc. 2003 IEEE Int. Frequency Control Symp. (IEEE, 2003) 704.

14 F. Lu, H. P. Lee, P. Lu, and S. P. Lim: Sens. Actuators, A 119 (2005) 90.

15 H. Sun, P. Lu, P. Zhang, and H. Chen: Proc. IEEE Sens. 2004 (IEEE, 2004) 95-98.

16 F. Lu, H. P. Lee, P. Lu, and S. P. Lim: Sens. Actuators, A 119 (2005) 90.

17 D. Puccio and E. P. EerNisse: Proc. 2008 the COMSOL Conf. (2008).

18 T. Sagawa, D. F. Wang, L. Jian, and R. Maeda: Proc. 2012 7th IEEE Int. Conf. Nano/Micro Engineered and Molecular Systems (NEMS) (IEEE, 2012) 591.

19 K. Wang, A. C. Wong, and C. T. C. Nguyen: J. Microelectromech. Syst. 9 (2000) 347.

20 M. Pandey, R. B. Reichenbach, A. T. Zehnder, A. Lal, and H. G. Craighead: J. Microelectromech. Syst. 18 (2009) 836.

21 J. Ji, H. Oigawa, M. Zhao, Y. Zhang, S. Ikezawa, and T. Ueda: IEEJ Trans. Sens. Micromach. 133 (2013) 253.

22 R. J. Besson: Proc. 1977 IEEE Int. Freq. Cont. Symp. (IEEE, 1977) 147. 


\section{About the Authors}

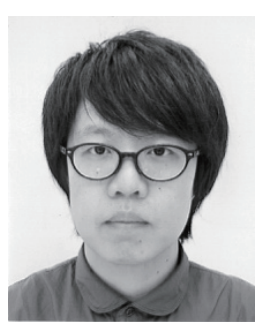

Jing Ji received his B.Sc. degree in microelectronic engineering from Nankai University, China, in 2003, and his M.S. and Ph.D. degrees in engineering of information, production and systems from the Graduate School of Information, Production and Systems, Waseda University, Japan, in 2007 and 2013, respectively. From 2013 to 2016, he worked as a research associate and researcher at the Information, Production and Systems Research Center, Waseda University, Japan. Since 2016, he has been an associate professor at Xidian University, P.R. China. He is a member of the Institute of Electrical and Electronics Engineers and the Institute of Electrical Engineers of Japan. His research area includes quartz MEMS devices, finite element method, and sensing technology.

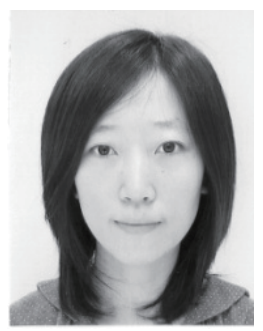

Meng Zhao received her B.Sc. degree in physics from Nankai University, China, in 2003. She received her Ph.D. degree in astrophysics from the Graduate University of the Chinese Academy of Sciences in 2009. From 2010 to 2015, She worked as a research associate and researcher at the Information, Production, and Systems Research Center, Waseda University, Japan. Since 2016, she has been an associate professor at Xidian University, P.R. China. She engages in the research and development of anisotropic wet etching simulators of quartz crystals.

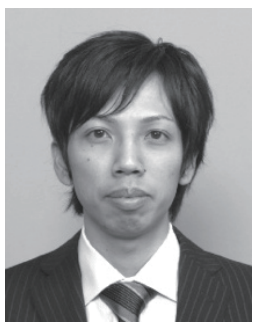

Hiroshi Oigawa received his M.E. degree from the Graduate School of Information, Production and Systems, Waseda University, Japan, in 2010. He is currently a Ph.D. candidate at the Graduate School of Information, Production and Systems, Waseda University. He is engaged in the research and development of quartz resonators and their applications to gas sensors.

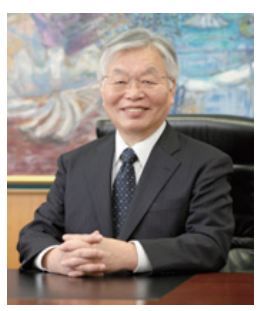

Toshitsugu Ueda received his B.E. and M.E. degrees in electrical engineering from Shinshu University, Nagano, Japan, in 1969 and 1971, respectively. He received his Ph.D. degree from Tokyo Institute of Technology in 1988. Since joining Yokogawa Electric Corporation in 1971, he has been engaged in developing low-noise amplifiers, mechanical resonators, micromachining technologies, and sensors using these technologies to control temperature, pressure, and displacement. He has been a professor of the Graduate School of Information, Production and Systems (IPS), Waseda University since 2003 and Dean of IPS and the IPS center in 2012. He received awards from the Society of Instrument and Control Engineers (Japan) in 1987 and 1994, as well as from the Japan Institute of Invention and Innovation in 1985 and 1987. He is a fellow of the Society of Instrument and Control Engineers (Japan). He is a member of the Institute of Electrical Engineers (Japan) and the Society of Instrument and Control Engineers (Japan). 